\title{
Significance of sTREM-1 in early prediction of ventilator-associated pneumonia in neonates: a single-center, prospective, observational study
}

Xingxing Zhao ${ }^{1+}$, Lixiao Xu ${ }^{2 \dagger}$, Zuming Yang ${ }^{1}$, Bin Sun ${ }^{3}$, Ying Wang ${ }^{3}, \mathrm{Gen} \mathrm{Li}^{2}$, Chenxi Feng ${ }^{2}$, Tao Pan ${ }^{3}$, Tian Yu ${ }^{2}$ and Xing Feng ${ }^{3^{*}}$

\begin{abstract}
Background: To evaluate whether soluble triggering receptor expressed on myeloid cells-1 (sTREM-1) can be used as an early predictor of ventilator-associated pneumonia (VAP).

Methods: Ventilated neonatal patients admitted into the neonatology department between January 2017 and January 2018 were divided into VAP $(n=30)$ and non-VAP $(n=30)$ groups. Serum sTREM, procalcitonin (PCT), Creactive protein and interleukin-6 levels were measured at 0, 24, 72, and $120 \mathrm{~h}$ after initiation of mechanical ventilation (MV). Correlations between blood biomarker concentrations and VAP occurrence were analyzed. Predictive factors for VAP were identified by logistic regression analysis and Hosmer-Lemeshow test, and the predictive value of sTREM-1 and biomarker combinations for VAP was determined by receiver operating characteristic curve analysis.
\end{abstract}

Results: The serum STREM-1 concentration was significantly higher in the VAP group than in the non-VAP group after 72 and $120 \mathrm{~h}$ of MV (72 h: 289.5 (179.6-427.0) vs 202.9 (154.8-279.6) pg/ml, $P<0.001 ; 120$ h: 183.9 (119.8-232.1) vs $141.3(99.8-179.1) \mathrm{pg} / \mathrm{ml}, P=0.042$ ). The area under the curve (AUC) for sTREM-1 at $72 \mathrm{~h}$ was 0.902 with a sensitivity of $90 \%$ and specificity of $77 \%$ for the optimal cut-off value of $165.05 \mathrm{pg} / \mathrm{ml}$. Addition of PCT to sTERM-1 at $72 \mathrm{~h}$ further improved the predictive value, with this combination having an AUC of 0.971 (95\% confidence interval: $0.938-1.000$ ), sensitivity of 0.96 , specificity of 0.88 , and Youden index of 0.84 .

Conclusion: STREM-1 is a reliable predictor of VAP in neonates, and combined measurement of serum levels of STREM-1 and PCT after $72 \mathrm{~h}$ of MV provided the most accurate prediction of VAP in neonatal patients.

Keywords: Neonate, Mechanical ventilation, Ventilator-associated pneumonia, Biomarker, Soluble myeloid cell trigger receptor-1

\footnotetext{
* Correspondence: xingfeng@suda.edu.cn

${ }^{+}$Xingxing Zhao and Lixiao Xu contributed equally to this work.

${ }^{3}$ Department of Neonatology, Children's Hospital of Soochow University, Suzhou 215000, China

Full list of author information is available at the end of the article
}

C C The Author(s). 2020 Open Access This article is licensed under a Creative Commons Attribution 4.0 International License, which permits use, sharing, adaptation, distribution and reproduction in any medium or format, as long as you give appropriate credit to the original author(s) and the source, provide a link to the Creative Commons licence, and indicate if changes were made. The images or other third party material in this article are included in the article's Creative Commons licence, unless indicated otherwise in a credit line to the material. If material is not included in the article's Creative Commons licence and your intended use is not permitted by statutory regulation or exceeds the permitted use, you will need to obtain permission directly from the copyright holder. To view a copy of this licence, visit http://creativecommons.org/licenses/by/4.0/ The Creative Commons Public Domain Dedication waiver (http://creativecommons.org/publicdomain/zero/1.0/) applies to the data made available in this article, unless otherwise stated in a credit line to the data. 


\section{Background}

Ventilator-associated complications have increasingly drawn attention in ventilated neonatal patients due to their detrimental impact on prognosis. Ventilatorassociated pneumonia (VAP) is one of the most commonly seen complications in neonatal patients [1], and it usually leads to a difficult ventilator weaning, which increases the duration of intensive care unit (ICU) stay [2]. The reported mortality rates among neonatal patients with VAP have been as high as $20-75 \%$ in both China and western countries [3-5]. Although early prediction of VAP could contribute to timely prevention and treatment, no effective predictors of VAP in neonates have been discovered so far [6]. The early diagnosis of VAP in neonates remains a challenge because clinical signs and radiological manifestations are non-specific, and tissue culture as the gold standard of diagnosis is timeconsuming, invasive and easily contaminated.

Several blood biomarkers have been tested in previous studies for their ability to aid the diagnosis and prognosis of VAP. Inflammatory cytokines such as interleukin-6 (IL-6), C-reactive protein (CRP), and procalcitonin (PCT) have clinical significance as predictive or prognostic markers of infection [7-12]. Specifically, PCT was shown to be effective for guiding antibiotic treatment [13]. The sensitivity and specificity of PCT for predicting the prognosis of VAP were superior to those of CRP, but both values were found to be effective predictors of the response to antibiotic therapy $[8,14]$. The serum IL6 concentration was shown to be correlated with the severity of VAP but with limited predictive value based on the finding that IL- 6 levels in serum and bronchoalveolar lavage fluid (BALF) did not differ significantly between patients with and without VAP [15]. Although studies of these biomarkers have provided important information regarding their value in VAP, the dynamic changes in the levels of these inflammatory biomarkers in neonates receiving mechanical ventilation (MV) have not been fully discovered and the possibility that a combination of these biomarkers could provide improved diagnostic or prognostic power in VAP has yet to be explored.

Myeloid cell trigger receptor-1 (TREM-1) is a member of the immunoglobulin super receptor family mainly expressed on the surface of myeloid cells such as neutrophils and monocytes/macrophages [16]. Soluble TREM1 (sTREM-1), one of the two forms of TREM-1, has been reported as a novel and strong indicator of pneumonia [17]. To date, studies of the predictive value of sTREM-1 in pneumonia patients have mainly enrolled adult patients, and the possible significance of the serum concentration of STREM-1 in neonates for the diagnosis and prognosis of VAP has not been investigated. Moreover, most studies have BALF samples to search for biomarkers for VAP. Importantly, sTREM-1 can be easily detected in blood samples, which are easier to obtain than BALF. The use of blood samples for measurement of biomarkers of VAP has been incompletely explored.

In this pilot study, we dynamically monitored the serum levels of sTREM-1 and the inflammatory cytokines PCT, CRP, and IL-6 in ventilated neonatal patients and investigated their diagnostic and predictive value for VAP.

\section{Methods}

\section{Patients and study design}

Patients admitted to the Neonatal Intensive Care Unit (NICU) at Children's Hospital of Soochow University from January 2017 to January 2018 were prospectively screened. All patients who were diagnosed with VAP were included. The diagnosis was determined according to the criteria for VAP in infants under 1 year old published by the US Centers for Disease Control and Prevention (CDC) and the National Hospital Safety Monitoring Network (NHSN) [1]. Briefly, VAP was defined by the presence of at least three clinical manifestations including new or persistent infiltration changes occurring in the lungs and gas exchange disorders after $48 \mathrm{~h}$ of MV and within $48 \mathrm{~h}$ after weaning. Other inclusion criteria included a length of MV longer than $120 \mathrm{~h}$ and the absence of systemic infection following MV.

The exclusion criteria included systemic severe infection, incomplete medical records, MV duration less than $120 \mathrm{~h}$, and surgical trauma.

All patients were ventilated with the MAQUET Servoi ventilator system (Germany) or SLE 5000 Infant Ventilator (United Kingdom). Chest X-ray was performed immediately after $\mathrm{MV}$, and routine X-ray was performed every 1-2 days thereafter.

The control group consisted of the same number of patients who received MV but did not develop VAP. The clinical characteristics of patients in the control group were well balanced with those of the VAP group in terms of gender, age, birth weight, gestational age, and disease severity.

\section{Sample collection and data recording}

Baseline characteristics including name, gender, gestational age, birth day and other general conditions were recorded. Clinical signs including temperature, apnea, and nasal agitation were dynamically monitored along with laboratory and imaging results.

Peripheral blood samples were collected at the start of MV (0 h) and after 24, 72 and $120 \mathrm{~h}$ of MV. Blood samples were centrifuged at $2000 \mathrm{rpm}$ for $5 \mathrm{~min}$ at room temperature, and the supernatant serum was collected and stored at $-80{ }^{\circ} \mathrm{C}$. The serum concentrations of sTREM-1 and IL-6 were measured using enzyme-linked 
immunosorbent assay (ELISA) kits according to the manufacturer's instructions (R\&D Systems Inc., USA). CPR and PCT were determined at the local hospital laboratory. CRP test was based on the principle of the latex agglutination and PCT was determined by immunofluorescence staining.

\section{Statistical analysis}

Normally distributed data were expressed as mean \pm standard deviation (SD) and compared between groups using the independent sample $t$ test. Non-normally distributed data were expressed as median with interquartile range and compared between groups using the Mann-Whitney U test. Categorical data were analyzed by the chi-square test or Fisher's exact test. Correlations between two variables were calculated by the Spearman test. A general linear model was established for age correction. The Hosmer-Lemeshow test was performed to evaluate the goodness of fit for the logistic regression model. Receiver operating characteristic (ROC) curves were plotted and employed in combination with the results from logistic regression analysis to evaluate the efficacy of a single index and combined indexes for VAP prediction. SPSS version 22.0 (SPSS, Inc., USA) was used for statistical processing, and GraphPad Prism 5 (GraphPad, USA) was used for preparation of graphs.

\section{Results}

\section{Patient characteristics}

Thirty pediatric patients who received MV for at least $120 \mathrm{~h}$ and developed VAP as well as 30 matched patients who did not develop VAP were included in the study. From the 112 cases initially enrolled, we excluded 61 patients for whom the duration of MV was less than $120 \mathrm{~h}$, 7 patients who had severe infection, 6 patients who had incomplete medical records and 8 patients who experienced surgical trauma. There were no significant differences in gender, mode of delivery, gestational age, birth weight, and underlying diseases between the VAP and non-VAP groups (Table 1).

\section{Serum sTREM-1 concentration in neonatal patients with and without VAP}

After initiation of MV, the serum sTREM-1 concentration was elevated, with peak values observed at $72 \mathrm{~h}$ and declining values observed thereafter in both groups (Fig. 1a). An increasing trend without statistical significance was noted from 0 and $24 \mathrm{~h}$ of $\mathrm{MV}$ in the VAP group and non-VAP group $(P=0.549$ and $P=0.112$, respectively). After 72 and $120 \mathrm{~h}$ of MV, the sTREM-1 levels in the VAP group were significantly increased to 289.5 (179.6-427.0) pg/ml and 183.9 (119.8-232.1) pg/ $\mathrm{ml}$, respectively, and these concentrations were significantly higher than those in the non-VAP group $(P<$ $0.001[<0.001$ after adjustment for age $]$ and $P=0.042$ $[P=0.040$ after adjustment for age; Fig. 1a, Supplemental Table 1). Comparisons of sTREM-1 levels in different timepoints were displayed in Supplementary Figure 1, which showed an obvious increase of sTREM-1 in VAP group at 72 and $120 \mathrm{~h}$.

\section{Dynamic changes in serum levels of inflammatory cytokines in patients with and without VAP}

The serum PCT levels followed a trend similar to that of the serum sTREM-1 concentration during MV (Fig. 1b). The serum PCT level was significantly greater after $72 \mathrm{~h}$ of $\mathrm{MV}$ in patients who developed VAP than in those who did not [1.89 (1.38-2.61) vs $1.10(0.75-1.36) \mathrm{pg} / \mathrm{ml}$, $P<0.001]$. The serum PCT level at $72 \mathrm{~h}$ in the VAP group was also significantly greater than those at 0,24 and $120 \mathrm{~h}$ (all $P<0.001$ ). The serum PCT levels after 24 and $120 \mathrm{~h}$ of $\mathrm{MV}$ appeared higher in the VAP group

Table 1 Baseline characteristics of neonatal patients in the VAP and non-VAP groups

\begin{tabular}{llll}
\hline Characteristics & VAP group $(n=30)$ & Non-VAP group $(n=30)$ & $P$ \\
\hline Gender (Male/Female) & $20 / 10$ & $21 / 9$ & 0.780 \\
Delivery mode (Natural/C-section) & $11 / 19$ & $9 / 21$ & 0.580 \\
Birth weight (g) & $2403 \pm 1073$ & $2628 \pm 912.4$ & 0.384 \\
Gestational age (weeks) & $33.78 \pm 3.807$ & $35.64 \pm 3.765$ & 0.063 \\
Age at admission (h) & $8.5(3.75-27)$ & $11.5(3.75-25)$ & 0.830 \\
Underlying diseases & 12 & 10 & 0.592 \\
RDS $(n=22)$ & 10 & 12 & 0.592 \\
Malformation $(n=22)$ & 4 & 3 & 1.000 \\
Pneumothorax $(n=7)$ & 2 & 3 & 1.000 \\
Asphyxiation $(n=5)$ & 2 & 1 & 1.000 \\
Pulmonary hemorrhage $(n=3)$ & 0 & 1 & 1.000 \\
MAS $(n=1)$ & & & \\
\hline
\end{tabular}




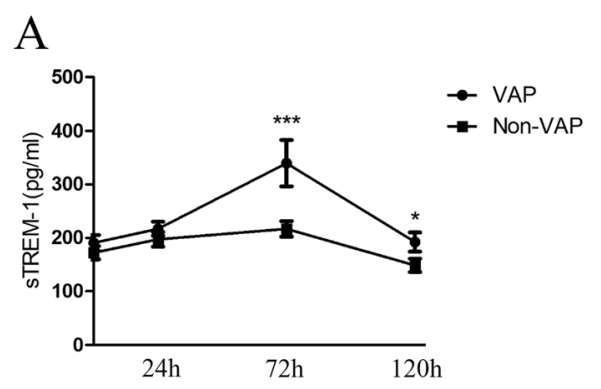

B
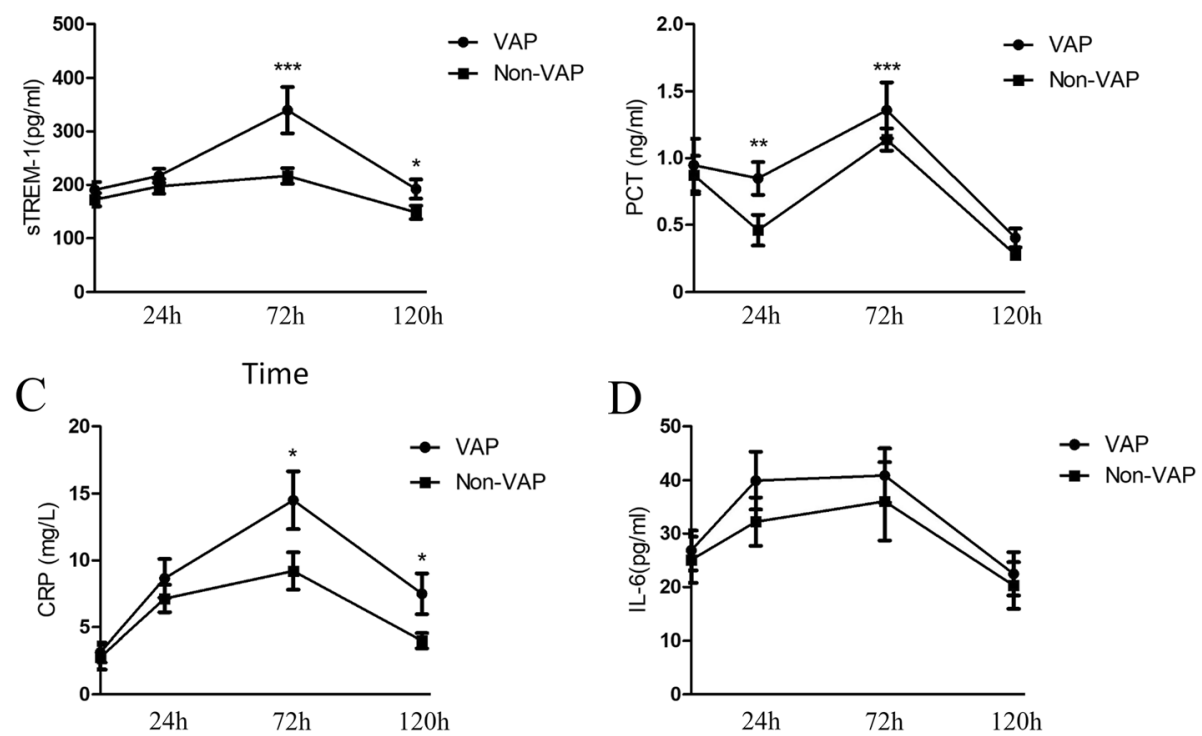

Fig. 1 Serum concentrations of sTREM-1, PCT, CRP and IL-6 over $120 \mathrm{~h}$ of MV in the VAP and non-VAP groups. ${ }^{*} P<0.05$ for VAP Vs. non-VAP group

than in the non-VAP group, but the differences were not significant after adjustment for age. The serum CRP level after $72 \mathrm{~h}$ of MV was also significantly greater in the VAP group than in the non-VAP group (10.06(6.8717.62) vs $7.97(4.43-12.56), P=0.047$ after adjustment for age; Fig. 1c). The serum IL-6 level did not differ between the two groups at any time point (Fig. 1d).

\section{Correlations between potential biomarkers and VAP}

Potential correlations between the measured biomarkers and VAP were analyzed at $0,24,72$ and $120 \mathrm{~h}$ of MV. A positive correlation was observed between the serum STREM-1 concentration at $72 \mathrm{~h}$ and VAP $(\mathrm{r}=0.697, P<$ 0.001). Positive correlations were also found between the serum PCT concentrations at 24 and $72 \mathrm{~h}$ and VAP $(\mathrm{r}=$ 0.429 and 0.601 , respectively, both $P<0.05$; Table 2 ).

\section{Predictive value of serum sTREM-1, PCT, CRP, and IL-6 for} VAP based on logistic regression analysis

The serum concentrations of sTREM-1, PCT, CRP and IL-6 at baseline and after $120 \mathrm{~h}$ of MV were not associated with the diagnosis of VAP. However, the serum

Table 2 Correlations between potential biomarkers and VAP

\begin{tabular}{|c|c|c|c|c|c|c|c|c|}
\hline \multirow[t]{2}{*}{ Biomarkers } & \multicolumn{2}{|l|}{$\mathrm{Oh}$} & \multicolumn{2}{|l|}{$24 \mathrm{~h}$} & \multicolumn{2}{|l|}{$72 \mathrm{~h}$} & \multicolumn{2}{|l|}{$120 \mathrm{~h}$} \\
\hline & $r$ & $P$ & $r$ & $P$ & $r$ & $P$ & $r$ & $P$ \\
\hline REM-1 & 0.079 & 0.549 & 0.208 & 0.111 & 0.697 & $<0.001$ & 0.266 & 0.040 \\
\hline PCT & -0.49 & 0.710 & 0.429 & 0.001 & 0.601 & $<0.001$ & 0.075 & 0.568 \\
\hline CRP & 0.035 & 0.793 & 0.042 & 0.748 & 0.260 & 0.045 & 0.269 & 0.038 \\
\hline L-6 & 0.166 & 0.204 & 0.135 & 0.305 & 0.204 & 0.118 & 0.162 & 0.217 \\
\hline
\end{tabular}

PCT concentration at $24 \mathrm{~h}$ was associated with VAP (odds ratio $[\mathrm{OR}] 2.724,95 \%$ confidence interval $[\mathrm{CI}]$ $1.021-7.270, P=0.045)$. At $72 \mathrm{~h}$, the serum concentrations of both PCT (OR 50.309, 95\%CI 4.045-625.720, $P=0.007)$ and sTREM-1 (OR 1.033, 95\%CI 1.009-1.057, $P=0.007)$ were significantly associated with VAP. The Hosmer-Lemeshow test showed that the $P$ value for the correlation between STREM-1 and VAP was greatest at $72 \mathrm{~h}(P=0.759>0.05)$, which implied a better degree of fitting.

ROC curve analysis of the predictive value of serum STREM-1, PCT, CRP and IL-6 for VAP

ROC curves for the ability of the serum sTREM-1, PCT, CRP and IL-6 at the four time points of MV to predict VAP were plotted (Fig. 2). The area under the curve (AUC) values were obviously greater after $72 \mathrm{~h}$ of $\mathrm{MV}$, and at this time point, the optimal cutoff value for the serum sTREM-1 concentration was $165.05 \mathrm{pg} / \mathrm{ml}$ with an AUC of 0.902 . The predictive sensitivity of sTREM-1 for VAP was $90 \%$ and the specificity was $77 \%$. Meanwhile, the AUC values were smaller for the serum PCT, CRP and IL6 concentrations (Fig. 2, Supplemental Table 2).

\section{ROC curves for the ability of a biomarker combination to predict VAP}

A ROC curve was constructed to examine the ability of the combination of serum sTREM-1 and PCT concentrations after $72 \mathrm{~h}$ of MV to predict VAP. The AUC, sensitivity, specificity and Youden index values were 0.971 (95\% CI: 0.938-1.000), 0.96, 0.88 and 0.84, respectively, and 

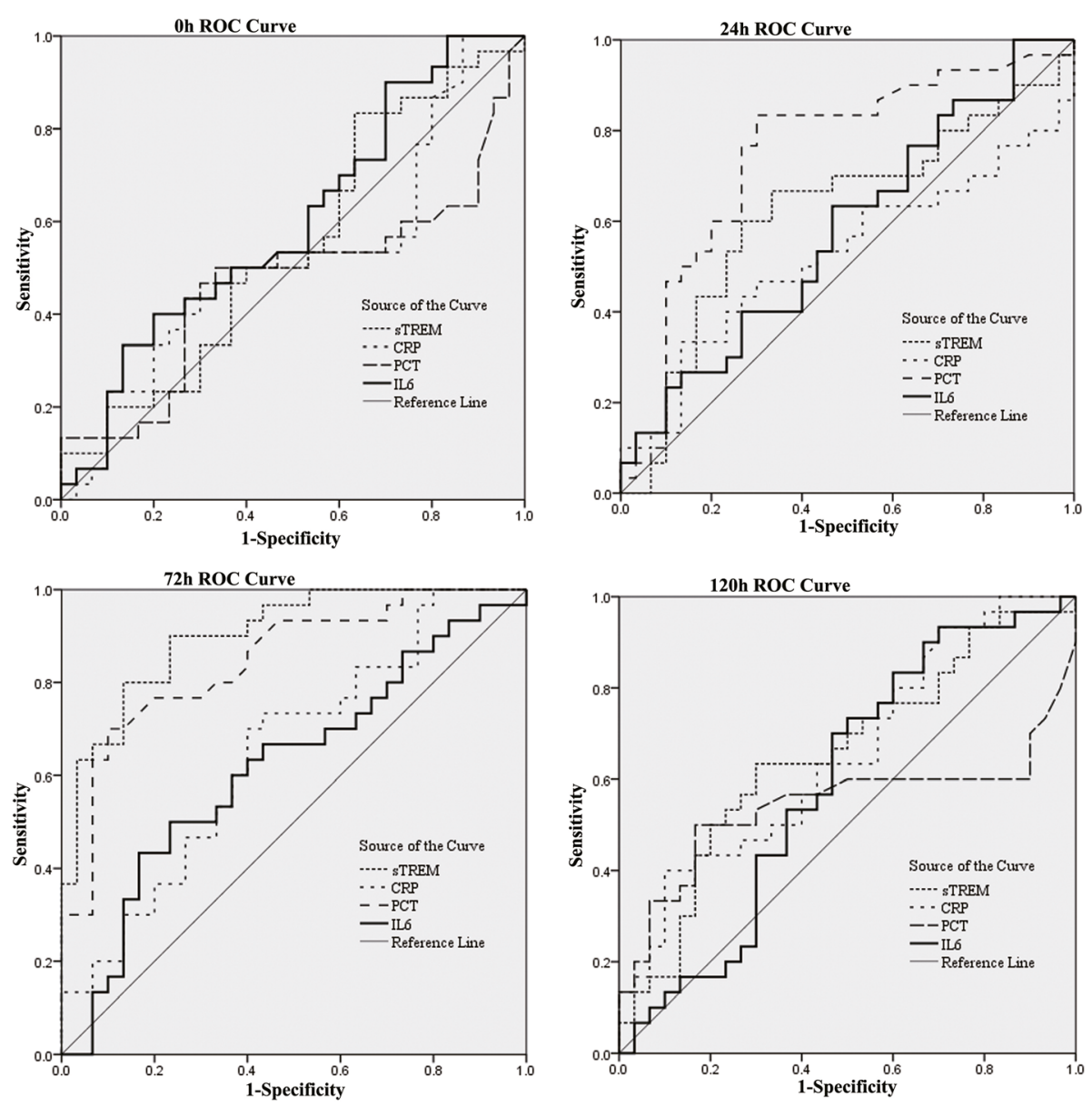

Fig. 2 ROC curves for the ability of the serum STREM-1, PCT, CRP and IL-6 concentrations at 0, 24, 72 and $120 \mathrm{~h}$ of MV to predict VAP

these values were all better than those for either single marker. The corresponding prediction equation was as follows: $P=1 / 1+\mathrm{e}^{-\mathrm{z}}$ 。 $\mathrm{Z}=0.027^{*}$ sTREM- $1+3.918^{*} \mathrm{PCT}$, where $P$ represents the predictive probability $(0 \leq P \leq 1)$ and $\mathrm{e}$ is the natural logarithm (Fig. 3).

\section{Discussion}

VAP is a frequently seen complication in neonates treated with $\mathrm{MV}$, and it is associated with a high morbidity and mortality $[2,18]$. Early recognition of VAP permits the timely implementations of treatment strategies [19], but unfortunately, early diagnosis of VAP is difficult mainly due to the lack of specific early clinical manifestations. The widely used approach of lower respiratory tract tissue culture is easily contaminated and may interfere with the diagnosis $[20,21]$. The current study evaluated the predictive value of the serum concentration of sTREM-1, in addition to other conventional biomarkers including PCT, CRP and IL-6, in the early detection of VAP. Our findings revealed that the serum sTREM-1 concentration after $72 \mathrm{~h}$ of MV could well predict the occurrence of VAP in neonatal patients, especially when combined with the serum PCT concentration at $72 \mathrm{~h}$ of MV. Conversely, the serum concentrations of CRP and IL- 6 did not exhibit solid predictive value for VAP in neonates.

sTREM-1 was reported to be a biomarker for the severity of infection [22]. In 2000, Bouchon A. et al. showed for the first time that TREM-1, as a member of the novel immunoglobulin super receptor family (Igsuperfamily, Ig-SF), triggers the release of chemokines and pro-inflammatory cytokines [23]. During acute infection, sTREM-1 is highly expressed and sheds from the cell surface into blood, saliva and other secretions. The dynamic blood level of sTREM-1 was shown to be associated with changes of disease severity [22]. In the current study, while the serum sTREM-1 concentrations were elevated in both groups of patients during the first $72 \mathrm{~h}$ of MV, patients in the VAP group had remarkably higher levels of sTREM-1 than those in the non-VAP group. MV alone can cause ventilator-associated lung injury (VALI) and enhance the release of multiple inflammatory cytokines. Therefore, it is not surprising that the sTREM-1 level was also increased in the non-VAP group 


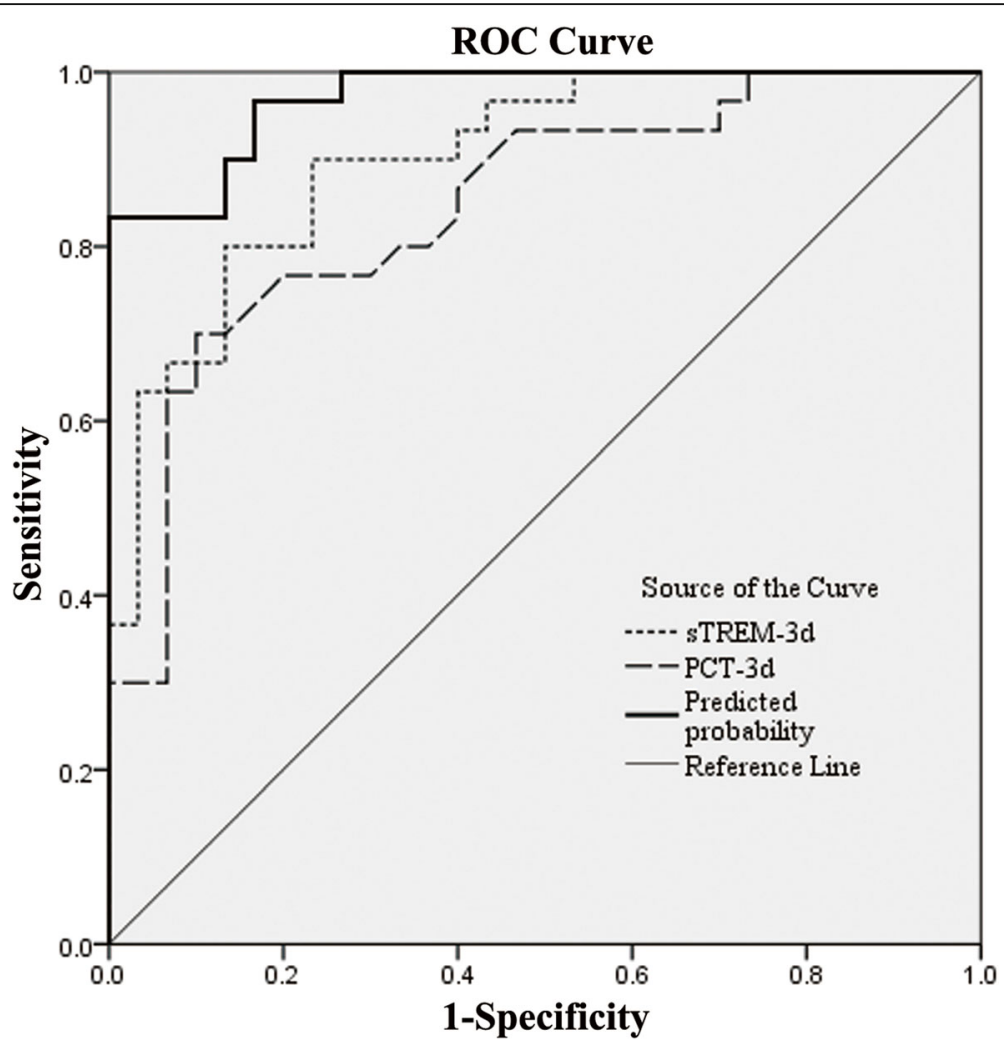

Fig. 3 ROC curve for the ability of the combination of STREM-1 and PCT to predict VAP

with MV. However, for patients with VAP, systemic infection may contribute to the even higher levels of sTREM-1 that we observed. Gibot et al. found that the increased level of sTREM-1 in septic patients decreased as the condition improved [24]. In the current study, a similar pattern in the serum sTREM-1 concentration was observed.

Previous studies of the predictive value of sTREM in lung disease have reported controversial results. sTREM1 was reported to be an independent predictive marker of pneumonia [24]. Another study evaluating the prognostic value of sTREM-1 in patients with lung diseases claimed that sTREM-1 is a powerful biomarker reflecting clinical outcome [25]. However, a conflicting result was reported by a previous study that assessed the predictive value of STREM-1 in pediatric patients with VAP and found no difference in serum and bronchoalveolar lavage sTREM-1 concentrations between the VAP and non-VAP groups [26]. There are several possible reasons for the discrepant findings between this previous study and our present study. First, the present study focused on neonates, whereas most previous studies only enrolled pediatric or adult patients. In addition, the underlying diseases could be different in different study populations, which might affect the study results. Also, the serum sTREM-1 concentrations were measured by
ELISA in the present study but determined by Western blotting in previous studies [27]. The use of different methods to measure sTREM-1 concentration might contribute to the disparity of the results. Moreover, different types of pathogens might affect the expression of sTREM-1. For example, bacterial and fungal infection can increase the expression of STREM-1, whereas almost no change in the sTREM-1 level has been observed in cases of viral infection [28]. Finally, the sample size was rather limited in the present study, and further research with a larger sample size is necessary.

Combined measurement of sTREM-1 and PCT showed more robust predictive performance for VAP in neonates. The AUC, sensitivity, specificity and Youden index for the combination of these markers were all better than those for either single marker. In previous studies, combined measurement of serum PCT and BALF sTREM-1 concentrations was reported to have potential for the detection of nosocomial sepsis and for discriminating VAP versus extrapulmonary infection or infection versus non-infectious disease [29, 30]. These previous findings support our observation that the combined measurements are preferable to a single marker for the prediction of VAP.

The present study has several limitations. First, this was a pilot study, and the sample size was small. 
However, the significant value of sTREM-1 for VAP prediction was still obvious. Second, all patients received antibiotics, and the effects of antibiotic treatment on the measurement results were not studied. Third, healthy neonates who did not require MV were not included in the study as a control group. Fourth, as we noticed, nonVAP group also had an increased level of sTREM-1 possibly caused by VALI. However, we did not perform bacterial or fungal cultures in non-VAP patients due to the study design. For adults, a previous study found that STREM-1 was present in a high concentration in the BALF of patients with bacterial VAP. However, the relationship between sTREM-1 level and microbial growth was not evaluated [31]. For neonates, obtaining a BALF specimen without contamination is often clinically infeasible partly because the neonate's airway is delicate. In addition, it's difficult to distinguish colonization from infection [32]. Fifth, the cut-off level of many inflammatory biomarkers may be depended on postnatal age. In addition, different measurement may also affect the cutoff level. However, due to the limited sample size, we did not further analyze the sTERM-1 level in patients with different postnatal age. Further large-scale studies might provide more information on the features of sTREM-1 in neonatal VAP.

In conclusion, the serum sTREM-1 concentration was found to be a reliable biomarker for the prediction of VAP in neonatal patients treated with $\mathrm{MV}$, and the combination of sTREM-1 and PCT concentrations showed even greater predictive power for VAP in these patients. Further large-scale multicenter trial is suggested to confirm our findings.

\section{Supplementary information}

Supplementary information accompanies this paper at https://doi.org/10. 1186/s12879-020-05196-z.

Additional file 1: Supplemental Table 1. Serum STREM-1 concentrations in neonatal patients in the VAP and non-VAP groups. MV: Mechanic ventilation; ${ }^{P}$ after adjustment for age

Additional file 2: Supplemental Table 2. Predictive performance of serum STREM-1, PCT, CRP and IL-6 concentrations at 0, 24, 72 and $120 \mathrm{~h}$ of MV AUC: area under the curve; Cl: confidence interval; PPV: positive predictive value; NPV: negative predictive value; PPR: positive probability ratio; NPR: negative probability ratio

Additional file 3: Supplementary Figure 1. Comparison of STREM-1 levels at $0,24,72$ and $120 \mathrm{~h}$ in VAP and non-VAP groups. ${ }^{*} P<0.05,{ }^{* * * *} P<0.001$

\footnotetext{
Abbreviations

AUC: Area under the curve; BALF: Bronchoalveolar lavage fluid; CRP: Creactive protein; CDC: Centers for Disease Control and Prevention; ELISA: Enzyme-linked immunosorbent assay; ICU: Intensive care unit; IL6: Interleukin-6; sTREM-1: Myeloid cells-1; MV: Mechanical ventilation; NICU: Neonatal Intensive Care Unit; NHSN: National Hospital Safety Monitoring Network; OR: Odds ratio; PCT: Procalcitonin; ROC: Receiver operating characteristic; SD: Standard deviation; VAP: Ventilator-associated pneumonia; VALI: Ventilator-associated lung injury
}

\section{Acknowledgements}

None.

\section{Authors' contributions}

ZXX and XLX had primary responsibility for the study design, data analysis and manuscript preparation. YZM participated in the sample collection and prepared the draft manuscript. SB and WY helped with clinical data analysis. LG and FCX helped in conducting the ELISA experiments. PT and YJ helped in the data analysis. FX contributed to the study design and provided valuable suggestions for conducting the experiments. All authors read and approved the final manuscript.

\section{Funding}

This work was supported by the National Natural Science Foundation of China (Nos. 81471488, 81671532, 81771625, 81871193); the Jiangsu Provincial Key Medical Discipline (No. ZDXKA2016013); the Jiangsu Province Key Research and Development of Special Funds in China (No. BE2015644); the Jiangsu Provincial Medical Youth Talentthe Jiangsu Provincial Medical Youth Talent (Nos. QNRC2016758, QNRC2016762, QNRC2016763); the Jiangsu Province Women and Children Health Research Project (No. F201750); the Public Health Technology Project of Suzhou City of China (Nos. SS201536, SYS201765); and the Department of Pediatrics Clinical Center of Suzhou City of China (No. Szzx201504). The funders had no role in study design, data collection and analysis, decision to publish, or preparation of the manuscript.

Availability of data and materials

The data and materials supporting the conclusions of the study are available from the corresponding author on reasonable request.

Ethics approval and consent to participate

This prospective study was approved by the ethics committee of Children's Hospital of Soochow University (No: 2016LW008), and written informed consent was obtained from each patient's legal guardians prior to treatment.

\section{Consent for publication}

The authors confirm that the work has not been published before or under consideration for publication elsewhere. The publication of this study has been approved by all co-authors.

\section{Competing interests}

The authors declare no conflicts of interest.

\section{Author details}

1Department of Neonatology, Suzhou Municipal Hospital, The Affiliated Suzhou Hospital of Nanjing Medical University, Suzhou 215000, China. ${ }^{2}$ Institute of Pediatric Research, Children's Hospital of Soochow University, Suzhou 215000, China. ${ }^{3}$ Department of Neonatology, Children's Hospital of Soochow University, Suzhou 215000, China.

Received: 29 July 2019 Accepted: 24 June 2020

Published online: 25 July 2020

\section{References}

1. Horan TC, Andrus M, Dudeck MA. CDC/NHSN surveillance definition of health care-associated infection and criteria for specific types of infections in the acute care setting. Am J Infect Control. 2008;36:309-32.

2. Pan $Y, D u L, A i ~ Q$, et al. Microbial investigations in throat swab and tracheal aspirate specimens are beneficial to predict the corresponding endotracheal tube biofilm flora among intubated neonates with ventilator-associated pneumonia. Exp Ther Med. 2017;14:1450-8.

3. Gokce IK, Kutman HGK, Uras N, Canpolat FE, Dursun Y, Oguz SS. Successful implementation of a bundle strategy to prevent ventilator-associated pneumonia in a neonatal intensive care unit. J Trop Pediatr. 2018;64:183-8.

4. Lee PL, Lee WT, Chen HL. Ventilator-associated pneumonia in low birth weight neonates at a neonatal intensive care unit: a retrospective observational study. Pediatr Neonatol. 2017:58:16-21.

5. Galal YS, Youssef MR, Ibrahiem SK. Ventilator-Associated Pneumonia: Incidence, Risk Factors and Outcome in Paediatric Intensive Care Units at Cairo University Hospital. J Clin Diagn Res. 2016;10:Sc06-11.

6. American Thoracic Society, Infectious Diseases Society of America. Guidelines for the management of adults with hospital- 
acquired,ventilatorassociated, and healthcare-associated pneumonia. Am J Respir CritCare Med. 2005;171:388-416.

7. Carey TS, Kinlaw A. Review: In primary care, CRP testing, shared decision making, and procalcitonin reduce antibiotic prescribing for ARI. Ann Intern Med. 2018;168:Jc11.

8. Tanriverdi $\mathrm{H}$, Tor MM, Kart L, Altin R, Atalay F, SumbSumbuloglu V. Prognostic value of serum procalcitonin and C-reactive protein levels in critically ill patients who developed ventilator-associated pneumonia. Ann Thorac Med. 2015;10:137-42.

9. Ramirez P, Ferrer M, Gimeno R, et al. Systemic inflammatory response and increased risk for ventilator-associated pneumonia: a preliminary study. Crit Care Med. 2009;37:1691-5.

10. Habib SF, Mukhtar AM, Abdelreheem HM, et al. Diagnostic values of CD64 $\mathrm{C}$-reactive protein and procalcitonin in ventilator-associated pneumonia in adult trauma patients: a pilot study. Clin Chem Lab Med. 2016;54:889-95.

11. Shelhamer MC, Rowan MP, Cancio LC, et al. Elevations in inflammatory cytokines are associated with poor outcomes in mechanically ventilated burn patients. J Trauma Acute Care Surg. 2015;79:431-6.

12. Salluh JIF, Souza-Dantas VC, Povoa P. The current status of biomarkers for the diagnosis of nosocomial pneumonias. Curr Opin Crit Care. 2017;23:391-7.

13. de Jong $E$, van Oers JA, Beishuizen A, et al. Efficacy and safety of procalcitonin guidance in reducing the duration of antibiotic treatment in critically ill patients: a randomised, controlled, open-label trial. Lancet Infect Dis. 2016;16:819-27.

14. Kiaei BA, Ghiasi F, Moradi D. Precalcitonin and C-reactive protein as markers in response to antibiotic treatment in ventilator-associated pneumonia in intensive care unit-hospitalized patients. Adv Biomed Res. 2015;4:240.

15. Chou CW, Lin FC, Tsai HC, Chang SC. The importance of pro-inflammatory and anti-inflammatory cytokines in pneumocystis jirovecii pneumonia. Med Mycol. 2013;51:704-12

16. Jolly L, Carrasco K, Derive M, Lemarie J, Boufenzer A, Gibot S. Targeted endothelial gene deletion of triggering receptor expressed on myeloid cells-1 protects mice during septic shock. Cardiovasc Res. 2018;114:907-18.

17. Gibot S, Cravoisy A, Levy B, Bene MC, Faure G, Bollaert PE. Soluble triggering receptor expressed on myeloid cells and the diagnosis of pneumonia. $\mathrm{N}$ Engl J Med. 2004;350:451-8.

18. Romo-Gamboa JP, Sandoval-Perez BA, Rodriguez-Lopez AB, Torres-Gonzalez MA, Barrera-de Leon JC. Risk factors related with ventilator-associated pneumonia in a neonatal intensive care therapy. Rev Med Inst Mex Seguro Soc. 2017:55(Suppl 1):S72-s79.

19. Schreiber MP, Shorr AF. Challenges and opportunities in the treatment of ventilator-associated pneumonia. Expert Rev Anti-Infect Ther. 2017;15:23-32.

20. Abo-Hagar HH, Abo-Elezz AA, Mehrez M, Mabrouk MM, Elshora OA. Diagnostic efficacy of serum amyloid a protein and soluble intercellular adhesion molecule 1 in pediatric ventilator-associated pneumonia. $J$ Intensive Care Med. 2019;34:503-10.

21. Brodska H, Valenta J, Pelinkova K, et al. Diagnostic and prognostic value of presepsin vs. established biomarkers in critically ill patients with sepsis or systemic inflammatory response syndrome. Clin Chem Lab Med. 2018;56: 658-68

22. Oku R, Oda S, Nakada TA, et al. Differential pattern of cell-surface and soluble TREM-1 between sepsis and SIRS. Cytokine. 2013;61:112-7.

23. Bouchon A, Dietrich J, Colonna M. Cutting edge: inflammatory responses can be triggered by TREM-1, a novel receptor expressed on neutrophils and monocytes. J Immunol. 2000;164:4991-5.

24. Gibot S. Soluble triggering receptor expressed on myeloid cells-1 and diagnosis of ventilator-associated pneumonia. Intensive Care Med. 2009;35: 1644 author reply 1645-1646.

25. Porfyridis I, Plachouras D, Karagianni V, et al. Diagnostic value of triggering receptor expressed on myeloid cells-1 and C-reactive protein for patients with lung infiltrates: an observational study. BMC Infect Dis. 2010;10:286.

26. Matsuno AK, Carlotti AP. Role of soluble triggering receptor expressed on myeloid cells-1 for diagnosing ventilator-associated pneumonia after cardiac surgery: an observational study. BMC Cardiovasc Disord. 2013;13:107.

27. Collins CE, La DT, Yang HT, et al. Elevated synovial expression of triggering receptor expressed on myeloid cells 1 in patients with septic arthritis or rheumatoid arthritis. Ann Rheum Dis. 2009;68:1768-74.

28. Huh JW, Lim CM, Koh Y, et al. Diagnostic utility of the soluble triggering receptor expressed on myeloid cells-1 in bronchoalveolar lavage fluid from patients with bilateral lung infiltrates. Crit Care. 2008;12:R6.
29. Cao C, Gu J, Zhang J. Soluble triggering receptor expressed on myeloid cell1 (sTREM-1): a potential biomarker for the diagnosis of infectious diseases. Front Med. 2017;11:169-77.

30. Schultz MJ, Determann RM. PCT and STREM-1: the markers of infection in critically ill patients? Med Sci Monit. 2008;14:Ra241-7.

31. Siranovic M, Kovac J, Gopcevic S, et al. Human soluble TREM-1: lung and serum levels in patients with bacterial ventilator associated pneumonia. Acta Clin Croat. 2011;50(3):345-9.

32. Cernada M, Brugada M, Golombek S, et al. Ventilator-associated pneumonia in neonatal patients: an update. Neonatology. 2014;105(2):98-107.

\section{Publisher's Note}

Springer Nature remains neutral with regard to jurisdictional claims in published maps and institutional affiliations.
Ready to submit your research? Choose BMC and benefit from:

- fast, convenient online submission

- thorough peer review by experienced researchers in your field

- rapid publication on acceptance

- support for research data, including large and complex data types

- gold Open Access which fosters wider collaboration and increased citations

- maximum visibility for your research: over $100 \mathrm{M}$ website views per year

At BMC, research is always in progress.

Learn more biomedcentral.com/submissions 